\title{
ON THE VANISHING PRIME GRAPH OF FINITE GROUPS
}

\author{
SILVIO DOLFI, EMANUELE PACIFICI, LUCIA SANUS, AND PABLO SPIGA
}

\begin{abstract}
Let $G$ be a finite group. An element $g \in G$ is called a vanishing element of $G$ if there exists an irreducible complex character $\chi$ of $G$ such that $\chi(g)=0$. In this paper we study the vanishing prime graph $\Gamma(G)$, whose vertices are the prime numbers dividing the orders of some vanishing element of $G$, and two distinct vertices $p, q$ are adjacent if and only if $G$ has a vanishing element of order divisible by $p q$. Among other things we prove that, similarly to what holds for the prime graph of $G$, the graph $\Gamma(G)$ has at most six connected components.
\end{abstract}

Keywords: finite groups, irreducible characters, prime graph.

\section{INTRODUCTION}

The prime graph of a finite group $G$, which in this paper we shall denote by $\Pi(G)$, is the simple undirected graph defined as follows: the vertices of $\Pi(G)$ are the prime numbers dividing the order of $G$, and two distinct vertices $p, q$ are adjacent if and only if $G$ has an element of order divisible by $p q$. This graph was introduced by K. Gruenberg and O. Kegel and, since then, several authors studied its nature and its influence on the group structure of $G$. For instance, it is known that $\Pi(G)$ has at most six connected components (see [16] or Theorem 6.3).

We focus our attention on a particular subgraph of $\Pi(G)$, which encodes nontrivial information arising from the set $\operatorname{Irr}(G)$ of irreducible complex characters of $G$. A vanishing element of $G$ is an element $g \in G$ such that $\chi(g)=0$ for some $\chi \in \operatorname{Irr}(G)$. Let $\operatorname{Van}(G)$ denote the set of vanishing elements of $G$. Now, the vanishing prime graph of $G$, denoted by $\Gamma(G)$, is the graph whose vertices are the prime divisors of the orders of the elements in $\operatorname{Van}(G)$, and two distinct vertices $p, q$ are adjacent if and only if $\operatorname{Van}(G)$ contains an element of order divisible by $p q$.

The vanishing prime graph was introduced in [4], where an investigation on $\Gamma(G)$ was made in the context of finite solvable groups. In particular, among other things, it was proved that $\Gamma(G)$ has at most two connected components in that case. (We refer to the Introduction of [4] for a more detailed discussion concerning this research area.) In the present paper, we extend this kind of investigation also to nonsolvable finite groups.

Theorem A. Let $G$ be a finite group. Then $\Gamma(G)$ has at most six connected components.

2000 Mathematics Subject Classification. 20C15.

The first and the second author are partially supported by the MIUR project "Teoria dei gruppi e applicazioni". The third author is partially supported by the Ministerio de Educación y Ciencia proyecto MTM2007-61161. 
Note that, as can be checked by direct inspection in [2], if $G$ is the sporadic simple group $J_{4}$, then we have $\Gamma(G)=\Pi(G)$ and $\Gamma(G)$ has six connected components. Therefore, the bound provided by the above theorem is best possible.

Theorem A is obtained using the following Theorem B. We denote by $\mathrm{n}(\mathcal{G})$ the number of connected components of a graph $\mathcal{G}$.

Theorem B. Let $G$ be a finite nonsolvable group. If $\Gamma(G)$ is disconnected, then $G$ has a unique nonabelian composition factor $S$, and $\mathrm{n}(\Gamma(G)) \leq \mathrm{n}(\Pi(S))$ unless $G$ is isomorphic to $A_{7}$.

The alternating group $A_{7}$ is a genuine exception to the above theorem, since it can be easily checked (in [2], for instance) that $\mathrm{n}\left(\Gamma\left(A_{7}\right)\right)=4$, whereas $\mathrm{n}\left(\Pi\left(A_{7}\right)\right)=3$. In fact, in Section 6 we shall see that $A_{7}$ is the unique nonabelian simple group $S$ such that $\Gamma(S) \neq \Pi(S)$.

A key tool in the proof of Theorem B is the following Theorem C, which describes the structure of a finite group $G$ such that a prime divisor of $|G|$ does not appear as a vertex of $\Gamma(G)$. We observe that Theorem $\mathrm{C}$ is a strengthening of [3, Corollary 3 ] and that its Part (b) is in turn a consequence of Theorem 3.1 of this paper.

Theorem C. Let $G$ be a finite group, $p$ a prime divisor of $|G|$, and $P$ a Sylow $p$-subgroup of $G$. If $G$ does not have any vanishing element of order divisible by $p$, then the following conclusions hold:

(a) $P$ is normal in $G$;

(b) either $G$ is abelian, or $G / \mathbf{O}_{p^{\prime}}(G)$ is a Frobenius group with kernel $P \mathbf{O}_{p^{\prime}}(G) / \mathbf{O}_{p^{\prime}}(G)$, and $\mathbf{O}_{p^{\prime}}(G)$ is nilpotent.

We prove Theorem C in Section 3, and Theorems A and B in Section 5. After that, in Section 6, we discuss some aspects of the relationship between the graphs $\Gamma(G)$ and $\Pi(G)$. In particular, we see that Theorem $\mathrm{A}$ and Theorem B also hold with $\Pi(G)$ in place of $\Gamma(G)$, but we present examples showing that the two graphs are significantly different in general.

We stress that our proofs of the results in this paper depend on the classification of the finite simple groups.

\section{Preliminary Results}

Throughout the whole paper, every group is assumed to be a finite group, and the set of prime divisors of the order of a group $G$ is denoted by $\pi(G)$.

In this section we present some preliminary results which will turn out to be useful in the sequel. We start by noticing that, by a classical theorem of W. Burnside, every nonlinear irreducible character vanishes on some group element. In particular, $\Gamma(G)$ is the empty graph if and only if $G$ is abelian.

In the following lemma we collect some basic remarks relating the vanishing elements of a group $G$ and the vanishing elements of the quotients of $G$. In particular, note that Lemma 2.1(a) shows that $\Gamma(G / N)$ is a subgraph of $\Gamma(G)$.

Lemma 2.1. Let $N$ be a normal subgroup of $G$.

(a) If $x N \in \operatorname{Van}(G / N)$, then $x N \subseteq \operatorname{Van}(G)$.

(b) Assume that $N$ has a complement $H$ in $G$, and let $A, B$ be subgroups of $H$. If $B \backslash A \subseteq \operatorname{Van}(H)$, then $B N \backslash A N \subseteq \operatorname{Van}(G)$. 
Proof. Every irreducible character of $G / N$ can be viewed, by inflation, as an irreducible character of $G$. So (a) follows immediately. Let now $\varphi: H \rightarrow G / N$ be the canonical isomorphism defined by $\varphi(x)=x N$, for $x \in H$. Then $\varphi(B) \backslash \varphi(A)=$ $(B N / N) \backslash(A N / N) \subseteq \operatorname{Van}(G / N)$, and hence $B N \backslash A N \subseteq \operatorname{Van}(G)$ by Part (a).

The next few results will play a role in the proof of Theorem $\mathrm{C}$.

Lemma 2.2. Let $M$ and $N$ be normal subgroups of $G$, and $L$ a subgroup of $N$. Assume that there exists $\psi \in \operatorname{Irr}(N)$ such that, for every $y \in G$ and $x \in L^{y} \backslash M$, we have $\psi(x)=0$. Then $L \backslash M \subseteq \operatorname{Van}(G)$.

Proof. Choose $\chi \in \operatorname{Irr}(G)$ lying over $\psi$. If $x \in L \backslash M$, then $\chi(x)=\chi_{N}(x)=$ $e \sum_{y \in R} \psi\left(x^{y}\right)$, for some subset $R$ of $G$ and some positive integer $e$. Since $x^{y} \in$ $L^{y} \backslash M$, we see that $\psi\left(x^{y}\right)=0$ for every $y \in R$. Hence $\chi(x)=0$ and $x \in \operatorname{Van}(G)$.

Let $G$ be a group, and let $V$ be a chief factor of $G$ (that is, there are two normal subgroups $A$ and $B$ of $G$ such that $V=A / B$ is a minimal normal subgroup of $G / B)$. In what follows, we denote by $\mathbf{C}_{G}(V)$ the subgroup $C$ of $G$ containing $B$ and such that $C / B=\mathbf{C}_{G / B}(V)$. Also, if $M$ is a normal subgroup of $G$, we say that $1=M_{0} \leq M_{1} \leq \cdots \leq M_{n}=M$ is a $G$-chief series of $M$ if it is part of a chief series of $G$ passing through $M$.

Lemma 2.3. Let $G$ be a group, and $V$ an abelian chief factor of $G$. Let $N \unlhd G$ be such that $N \mathbf{C}_{G}(V) / \mathbf{C}_{G}(V)$ is abelian. Then $N \backslash \mathbf{C}_{G}(V) \subseteq \operatorname{Van}(G)$.

Proof. Let $A$ and $B$ be normal subgroups of $G$ such that $V=A / B$. Replacing $G$ with the quotient group $G / B$, it is easily seen that we can assume $B=1$. So $V$ is a minimal normal subgroup of $G$ and it can be regarded as a simple $G$-module over a field of $p$ elements, for a suitable prime $p$. Set $\bar{G}=G / \mathbf{C}_{G}(V)$, and adopt the bar convention. By Clifford's theorem, $V$ is a semisimple (and faithful) $\bar{N}$-module. This forces $\mathbf{O}_{p}(\bar{N})$ to be trivial, and therefore, as $\bar{N}$ is abelian, we get $(|\bar{N}|,|V|)=1$. As is well known, it follows that there exists a regular orbit in the action of $\bar{N}$ on the elements of $V$. Applying Glauberman correspondence ([6, Theorem 18.9]), there exists $\phi \in \operatorname{Irr}(V)$ such that the inertia subgroup $I_{\bar{N}}(\phi)$ of $\phi$ in $\bar{N}$ is trivial, whence $I_{N}(\phi)=N \cap \mathbf{C}_{G}(V)$.

Now, let $\chi$ be an irreducible character of $G$ lying over $\phi$. By Clifford Correspondence we have $\chi=\psi^{G}$, where $\psi$ is an irreducible character of $I=I_{G}(\phi)$. For every $g$ in $G$, we get

$$
I^{g} \cap N=(I \cap N)^{g}=I_{N}(\phi)^{g} \leq \mathbf{C}_{G}(V)^{g}=\mathbf{C}_{G}(V) .
$$

As $\chi$ vanishes in $G \backslash \bigcup_{g \in G} I^{g}$, the desired conclusion follows.

Lemma 2.4. Let $M \leq N \leq G$, with $M$ and $N$ normal in $G$ and $(|M|,|N / M|)=1$. Assume that $M$ is minimal normal in $G$ or that $M$ is nilpotent. If $\mathbf{C}_{N}(M) \leq M$ and $N / M$ is abelian, then $N \backslash M \subseteq \operatorname{Van}(G)$.

Proof. If $M$ is minimal normal in $G$, then this is [3, Lemma 2.9]. So, assume that $M$ is nilpotent. Let $1=M_{0} \leq M_{1} \leq \cdots \leq M_{n}=M$ be a $G$-chief series of $M$. Then each factor $V_{i}=M_{i} / M_{i-1}, i=1,2, \ldots, n$, is an abelian chief factor of $G$. Also, since $M$ is nilpotent, it follows that $M$ lies in $C=\bigcap_{i=1}^{n} \mathbf{C}_{N}\left(V_{i}\right)$, whence $N / \mathbf{C}_{N}\left(V_{i}\right)$ is abelian for all $i \in\{1, \ldots, n\}$. By Lemma 2.3 it is then enough to show that $C$ actually coincides with $M$. In fact, let $H$ be a complement for $M$ in $N$ : if $h \in H$ 
centralizes every factor $V_{i}$ then, by coprimality, $h \in \mathbf{C}_{N}(M) \leq M$ and therefore $h=1$. Now, by Dedekind's law, we get $C=M(C \cap H) \leq M$, and the result follows.

Proposition 2.5. Let $G$ be a group, and let $M$ and $N$ be normal subgroups of $G$ such that $M \cap N=1$. Assume that there is an element $m \in M \cap \operatorname{Van}(G)$. Then $m n \in \operatorname{Van}(G)$ for all $n \in N$.

Proof. Let $\chi$ be an irreducible character of $G$ such that $\chi(m)=0$, and let $\alpha$ be an irreducible constituent of $\chi_{M}$. We get

$$
0=\chi(m)=e \sum_{i=1}^{s} \alpha^{g_{i}}(m)
$$

where $e=\left\langle\chi_{M}, \alpha\right\rangle \neq 0$, and $\left\{g_{1}, \ldots, g_{s}\right\}$ is a right transversal for the inertia subgroup $I_{G}(\alpha)$ of $\alpha$ in $G$.

Consider now the irreducible character $\alpha \times 1_{N}$ of $M N$. It is easy to check that $I_{G}\left(\alpha \times 1_{N}\right)=I_{G}(\alpha)$. Let $\gamma$ be an irreducible character of $I_{G}\left(\alpha \times 1_{N}\right)$ lying over $\alpha \times 1_{N}$, and set $\psi=\gamma^{G}$. Notice that, by Clifford Correspondence, we get $\psi \in \operatorname{Irr}(G)$. Then, setting $f=\left\langle\psi_{M N}, \alpha \times 1_{N}\right\rangle$, for every $n$ in $N$ we have

$$
\psi(m n)=f \sum_{i=1}^{s}\left(\alpha \times 1_{N}\right)^{g_{i}}(m n)=f \sum_{i=1}^{s} \alpha^{g_{i}}(m)=0,
$$

and $m n$ is in $\operatorname{Van}(G)$, as claimed.

Corollary 2.6. Let $G$ be a group and $K$ a nilpotent normal subgroup of $G$. If $K \cap \operatorname{Van}(G) \neq \emptyset$, then there exists $g \in K \cap \operatorname{Van}(G)$ whose order is divisible by every prime in $\pi(K)$.

Proof. Let $m$ be in $K \cap \operatorname{Van}(G)$ and $\pi$ the set of primes dividing $o(m)$. Let $N$ be the Hall $\pi^{\prime}$-subgroup of $K$. By Proposition 2.5, it suffices to take $g=m n$, where $n \in N$ has order divisible by every prime in $\pi^{\prime}$ (if any).

Let $p$ be a prime number, and $\chi$ an irreducible character of $G$. We recall that $\chi$ is said to be of $p$-defect zero if $p$ does not divide $|G| / \chi(1)$. By a well-known result of R. Brauer ([9, Theorem 8.17]), if $\chi$ is an irreducible character of $p$-defect zero of $G$ then, for every $g \in G$ such that $p$ divides the order $o(g)$, we have $\chi(g)=0$. In view of that, irreducible characters of $p$-defect zero appear to be relevant when dealing with vanishing elements.

In particular, the following Lemma 2.7 will turn out to be useful for our purposes.

Lemma 2.7. Let $G$ be a group, $N$ a normal subgroup of $G$, and $p$ a prime divisor of $|N|$. If $N$ has an irreducible character of p-defect zero, then every element of $N$ of order divisible by $p$ is a vanishing element of $G$.

Proof. Take $\psi \in \operatorname{Irr}(N)$ of $p$-defect zero, and choose $\chi \in \operatorname{Irr}(G)$ lying over $\psi$. By Clifford's theorem, $\chi_{N}$ is a sum of $G$-conjugates $\psi_{i}$ of $\psi$. As $\psi_{i}(1)=\psi(1)$, every $\psi_{i}$ is an irreducible character of $p$-defect zero of $N$. Hence every $\psi_{i}$ vanishes on every element of $N$ of order divisible by $p$. If now $x$ is such an element, we get $\chi(x)=\sum_{i} \psi_{i}(x)=0$, whence $x \in \operatorname{Van}(G)$.

Next, we recall a theorem from [5], which provides significant information concerning the existence of irreducible characters of $p$-defect zero for nonabelian simple 
groups. Also this result, together with the subsequent corollary, will turn out to be useful in the proof of Theorem C.

Theorem 2.8. ([5, Corollary 2]). Let $S$ be a nonabelian simple group, and $p$ a prime divisor of $|S|$. Then $S$ has an irreducible character of $p$-defect zero unless one of the following holds.

(a) The prime $p$ is 2 , and $S$ is isomorphic to either $M_{12}, M_{22}, M_{24}, J_{2}, H S$, Suz, $R u, C o_{1}, C o_{3}, B M$ or $A_{n}$, where $n \neq 2 m^{2}+m$ and $n \neq 2 m^{2}+m+2$ for every integer $m$.

(b) The prime $p$ is 3 , and $S$ is isomorphic to either $S u z, C o_{3}$ or $A_{n}$, where $3 n+1=$ $m^{2} d$ with d squarefree and divisible by some prime $r \equiv 2(\bmod 3)$.

Corollary 2.9. Let $G$ be a group, $N$ a nonabelian minimal normal subgroup of $G$, and $p$ a prime divisor of $|N|$. If $p \geq 5$, then every element of $N$ having order divisible by $p$ is a vanishing element of $G$. In particular, $N \cap \operatorname{Van}(G) \neq \emptyset$.

Furthermore, if $N$ is not a simple group then, for every $q \in \pi(N)$ with $q \neq p$, there exists $g \in \operatorname{Van}(G) \cap N$ of order divisible by $p q$.

Proof. Write $N=S_{1} \times \cdots \times S_{n}$, where $S_{i} \simeq S$ and $S$ is a nonabelian simple group whose order is divisible by $p$. As $p \geq 5$, by Theorem 2.8 there exists an irreducible character $\theta$ of $S$ of $p$-defect zero. Let $\psi=\theta \times \cdots \times \theta \in \operatorname{Irr}(N)$. Since $\psi$ is clearly a character of $p$-defect zero of $N$, the first claim of the statement follows from Lemma 2.7. Moreover, by Burnside's $p^{\alpha} q^{\beta}$-theorem, there certainly exists a prime divisor $p \geq 5$ of $|N|$. Hence, $N \cap \operatorname{Van}(G) \neq \emptyset$.

For the second claim, assume $n>1$. Let $x \in S_{1}$ be of order $p$, and let $q \in \pi(N)$ with $q \neq p$. As $q \in \pi\left(S_{2}\right)$, we can choose $y \in S_{2}$ of order $q$. Now $g=x y \in N$ is an element of order $p q$, and therefore $g \in \operatorname{Van}(G) \cap N$.

In the proof of Theorem B, we shall take advantage of some information concerning characters of sporadic simple groups and alternating groups. These information, yielded by Proposition 2.10, will be useful when dealing with nonabelian simple groups which fail to have irreducible characters of $p$-defect zero for some prime $p$, i.e. groups in the list of Theorem 2.8.

The character table of symmetric and alternating groups is well known (see for instance [11]). In particular, there exists a natural bijection between the irreducible characters of $S_{n}$ and the partitions of $n$. Also, given the partition $\pi$ of $n$ corresponding to the character $\chi$ of $\operatorname{Irr}\left(S_{n}\right)$, we have that $\chi_{A_{n}}$ is irreducible if and only if the Ferrers-Young diagram of $\pi$ is not self-associate, i.e. symmetric. In the proof of Proposition 2.10 we make use of the Murnaghan-Nakayama formula ([11, 2.4.7]). Also, we denote by $\chi_{\pi}$ the irreducible character of $S_{n}$ corresponding to the partition $\pi$ of $n$ and we identify (up to conjugation) an element of $S_{n}$ with a partition of $n$, i.e. its "cycle type" ([11, 1.2.4]).

Proposition 2.10. Let $S$ be a sporadic simple group, or an alternating group on $n$ letters with $n \geq 8$. Then $S$ has an irreducible character $\chi$ which extends to $\operatorname{Aut}(S)$ and an element $g$ of order 6 such that $\chi(g)=0$.

Proof. As regards sporadic simple groups, the claim is easily proved by direct inspection (see [2]). Therefore, we shall assume $S \simeq A_{n}$, and we aim to produce a partition $\pi$, together with an element $g \in S$, such that the corresponding character $\chi_{\pi}$ and $g$ satisfy the desired conditions. 
Set $m=n-4$. If $m \equiv 2 \bmod 3$, then take $\pi=(m, 3,1)$ and $g=\left(3^{(m-2) / 3}, 2^{2}, 1^{2}\right)$. If $m \equiv 1 \bmod 3$ and $m \neq 4$, then take $\pi=\left(m, 2,1^{2}\right)$ and $g=\left(3^{(m-1) / 3}, 2^{2}, 1\right)$. If $m=4$, then take $\pi=(4,4)$ and $g=(6,2)$. If $m \equiv 0 \bmod 3$, then take $\pi=\left(m-1,3,1^{2}\right)$ and $g=\left(3^{m / 3}, 2^{2}\right)$.

We close this preliminary section with some remarks concerning Frobenius groups. Recall that a Frobenius group is a product $G=F H$ with $F \unlhd G, 1<H<G$, $F \cap H=1$ and such that two elements $x \in F$ and $y \in H$ commute only if $x=1$ or $y=1$ (i.e. $H$ acts fixed-point freely on $F$ by conjugation). In this setting, $F$ is called the kernel and $H$ a complement of $G$. A classical result, Thompson's nilpotency criterion, implies that $F$ is nilpotent (see for instance [7, V.8.14]). Moreover, every subgroup of $H$ of order the product of two (possibly equal) primes is cyclic and every Sylow subgroup of $H$ is either cyclic or a generalized quaternion group. Finally, by a theorem of Zassenhaus, either $H$ is solvable or $H$ has a unique nonsolvable composition factor, which is isomorphic to $A_{5}$ (see $\left.[6,16.7 \mathrm{~d})\right]$ ).

In the following lemma, we gather some information on $\Gamma(G)$ when $G$ is a Frobenius group.

Lemma 2.11. Let $G$ be a Frobenius group with kernel $F$ and complement $H$. The graph $\Pi(G)$ has two connected components, whose vertex sets are $\pi(F)$ and $\pi(H)$. If $F \cap \operatorname{Van}(G) \neq \emptyset$, then we have $\Gamma(G)=\Pi(G)$, otherwise $\Gamma(G)$ coincides with the connected component of $\Pi(G)$ with vertex set $\pi(H)$.

Proof. By the structure of Frobenius groups, every nonidentity element of $G$ is either a $\pi(F)$-element or a $\pi(H)$-element, and $\pi(F) \cap \pi(H)=\emptyset$. Since $F$ is nilpotent, the primes in $\pi(F)$ are vertices of $\Pi(G)$ which are pairwise adjacent. Also the primes in $\pi(H)$ lie in the same connected component of $\Pi(G)$ : in fact, [7, V.8.18 c)] guarantees that $\mathbf{Z}(H)$ is not trivial. So, if $q$ is a prime divisor of $|\mathbf{Z}(H)|$, it is easily seen that every other prime divisor of $|H|$ is adjacent to $q$ in $\Pi(G)$. By the character theory of Frobenius groups, we get $G \backslash F \subseteq \operatorname{Van}(G)$. Therefore, the rest of the lemma follows from Corollary 2.6.

It may be worth mentioning that both the situations outlined for $\Gamma(G)$ in the conclusions of Lemma 2.11 can occur. An example of the latter situation is $S_{3}$, whereas an infinite family of examples of the former is provided in [1, Example 2].

\section{Proof of Theorem C}

Theorem $\mathrm{C}$ can be derived as a consequence of the following result, which may be of independent interest.

Theorem 3.1. Let $G$ be a group, and $p$ a prime divisor of $|G|$. Assume that $G$ has a normal Sylow $p$-subgroup $P$. If, for every prime $q \neq p$, there is no vanishing element of $G$ of order divisible by $p q$, then $G / \mathbf{O}_{p^{\prime}}(G)$ is either a p-group, or a Frobenius group with kernel $P \mathbf{O}_{p^{\prime}}(G) / \mathbf{O}_{p^{\prime}}(G)$. Moreover, $\mathbf{O}_{p^{\prime}}(G)$ is nilpotent.

Proof. We note that the assumptions are preserved by factoring out the normal subgroup $\mathbf{O}_{p^{\prime}}(G)$. Thus, we shall first assume $\mathbf{O}_{p^{\prime}}(G)=1$ and prove that, if $G>P$, then $G$ is a Frobenius group with kernel $P$.

By the Schur-Zassenhaus theorem, there exists a complement $H>1$ for $P$ in $G$, and this $H$ acts faithfully (by conjugation) on $P$ because $\mathbf{O}_{p^{\prime}}(G)=1$. Set $F$ to be the Fitting subgroup of $H$. We will go through a few steps. 
(i) If $A$ is an abelian normal subgroup of $H$, then $A P \backslash P \subseteq \operatorname{Van}(G)$.

In fact, $A P \unlhd G$ and then $\mathbf{C}_{A P}(P) \leq P$ because by assumption $\mathbf{O}_{p^{\prime}}(G)$ is trivial. Hence, by Lemma 2.4, we get $A P \backslash P \subseteq \operatorname{Van}(G)$.

(ii) $Q P \backslash P \subseteq \operatorname{Van}(G)$, for every Sylow subgroup $Q$ of $F$.

Let $q$ be a prime, and $Q$ a Sylow $q$-subgroup of $F$. Also, let $A$ be a characteristic abelian subgroup of $Q$. Now, step $(i)$ yields $A P \backslash P \subseteq \operatorname{Van}(G)$. As $p q$ does not divide the order of any vanishing element of $G$, the prime $p$ does not divide $o(g)$ for every $g \in A P \backslash P$. Hence $A$ acts fixed-point freely on $P$ and, since $A$ is abelian, we get that $A$ is cyclic.

The paragraph above shows that every characteristic abelian subgroup of $Q$ is cyclic. Recalling [14, Theorem 1.2], we can write $Q=E T$, with $E \cap T=Z \leq \mathbf{Z}(Q)$, $|Z|=q$, and $[E, T]=1$. The group $E$ is extraspecial or $E=Z$. There exists a cyclic subgroup $U \leq T$ with $|T: U| \leq 2$ and $U=\mathbf{C}_{T}(U)$. Further, $U$ and $E U$ are characteristic subgroups of $Q$.

In the case when $E \neq Z$, we have $(E U)^{\prime}=Z$ and $U=\mathbf{Z}(E U)$, whence, by [6, Theorem 7.5], every nonlinear irreducible character of $E U$ vanishes on $E U \backslash U$. As $Z \leq U$, clearly the same conclusion vacuously holds if $E=Z$.

Thus, by Lemma 2.1 and Lemma 2.2, we have $E U P \backslash U P \subseteq \operatorname{Van}(G)$. Moreover, step $(i)$ yields $U P \backslash P \subseteq \operatorname{Van}(G)$. Therefore, $E U P \backslash P \subseteq \operatorname{Van}(G)$.

If $q \neq 2$ we get $Q=E U$, therefore $Q P=E U P$, and $(i i)$ is proved. So, we can assume $q=2$.

Recalling that no vanishing element of $G$ has order divisible by $p q$, it follows that $\mathbf{C}_{E U}(x)=1$ for every nontrivial $x \in P$. Hence $E U P$ is a Frobenius group with kernel $P$. This implies that $E U$ is either cyclic or generalized quaternion. Assume first that $E U$ is generalized quaternion. Since $E U / Z$ is abelian, and since the index of the derived subgroup of a generalized quaternion group is 4 , it follows that $E U=E \simeq Q_{8}$, and $U=Z$. In particular, $T=\mathbf{C}_{T}(Z)=\mathbf{C}_{T}(U)=U$. Hence, also in this case we get $Q=E U$, and (ii) is proved.

Finally, if $E U$ is cyclic, then $E U=U$ and $Q=T$. Now, $U$ is an abelian normal subgroup of index at most 2 in $Q$, so if $\psi$ is a nonlinear irreducible character of $Q$, then $\psi$ vanishes on $Q \backslash U$ (see for instance [9, Lemma 2.29]). Thus, by Lemma 2.2 and Lemma 2.1, it follows that $Q P \backslash U P \subseteq \operatorname{Van}(G)$. Hence, as $U P \backslash P \subseteq \operatorname{Van}(G)$, we get that $Q P \backslash P \subseteq \operatorname{Van}(G)$ and $(i i)$ is proved.

(iii) $F P \backslash P \subseteq \operatorname{Van}(G)$.

Let $Q$ be a Sylow $q$-subgroup of $F$. Since no vanishing element of $G$ has order divisible by $p q$, step $(i i)$ yields $\mathbf{C}_{Q}(x)=1$, for every nontrivial $x \in P$. Thus, $Q$ acts fixed-point freely on $P$. It follows that $Q$ is either cyclic or generalized quaternion.

Therefore, $F=C \times D$ with $C$ cyclic of odd order and $D$ either cyclic or generalized quaternion. Define a subgroup $U$ of $F$ as follows: $U=F$ if $D$ is cyclic, $U=\mathbf{Z}(F)$ if $D \simeq Q_{8}$, and $U$ is the characteristic subgroup of index 2 in $F$ if $D \simeq Q_{2^{k}}$, for $k \geq 4$.

Hence, $U$ is a cyclic characteristic subgroup of $F$. So, $U \unlhd H$ and hence $U P \backslash P \subseteq$ $\operatorname{Van}(G)$ by step $(i)$. Moreover, if $U \neq F$, then every nonlinear irreducible character of $F$ vanishes on $F \backslash U$. Namely, if $D \simeq Q_{2^{k}}$ with $k \geq 4$, then this follows from [9, Lemma 2.29]. Also, if $D \simeq Q_{8}$, then this follows by direct inspection. Thus, Lemma 2.2 yields $F \backslash U \subseteq \operatorname{Van}(H)$ and then $F P \backslash U P \subseteq \operatorname{Van}(G)$ by Lemma 2.1. Therefore, $F P \backslash P \subseteq \operatorname{Van}(G)$. 
In the sequel, let $1=M_{0} \leq M_{1} \leq \cdots \leq M_{n}=F$ be an $H$-chief series of $F$ and write $V_{i}=M_{i} / M_{i-1}$ for $i=1, \ldots, n$. Define

$$
L=\bigcap_{i=1}^{n} \mathbf{C}_{H}\left(V_{i}\right),
$$

the centralizer in $H$ of all the chief factors $V_{i}$. Observe that $F \leq L \unlhd H$.

(iv) $G \backslash L P \subseteq \operatorname{Van}(G)$.

Recall that $F=C \times D$, with $C$ cyclic of odd order and $D$ either cyclic or generalized quaternion. If $D$ is cyclic, then $F$ has a characteristic series whose factors are cyclic of prime order. Indeed, the same conclusion holds also if $D \nsucceq Q_{8}$, as in this case $F$ has a unique cyclic subgroup of index 2. If $D \simeq Q_{8}$, then $F$ has a characteristic series whose factors are cyclic of prime order or isomorphic to $C_{2} \times C_{2}$. Therefore, in any case, every $H$-chief factor of $F$ is cyclic or isomorphic to $C_{2} \times C_{2}$. Let now $V$ be any of these factors and let $A=H / \mathbf{C}_{H}(V)$. We claim that $H \backslash \mathbf{C}_{H}(V) \subseteq \operatorname{Van}(H)$.

If $V$ is cyclic, then $A$ is abelian, and an application of Lemma 2.3 yields that $H \backslash \mathbf{C}_{H}(V) \subseteq \operatorname{Van}(H)$.

If $V \simeq C_{2} \times C_{2}$, then $A$ is isomorphic to a subgroup of $S_{3}$. If $A$ has order less than 6, then we can argue as in the paragraph above, getting $H \backslash \mathbf{C}_{H}(V) \subseteq \operatorname{Van}(H)$. In the case when $A$ is isomorphic to $S_{3}$, the group $A$ has an abelian normal subgroup $U / \mathbf{C}_{H}(V)$ of index 2. By Lemma 2.3, we have $U \backslash \mathbf{C}_{H}(V) \subseteq \operatorname{Van}(H)$. Moreover, the unique nonlinear irreducible character of $A$ vanishes on every element outside $U / \mathbf{C}_{H}(V)$, whence such elements are in $\operatorname{Van}(A)$ and, by Lemma 2.1, we see that $H \backslash U \subseteq \operatorname{Van}(H)$. Therefore, also in this case $H \backslash \mathbf{C}_{H}(V) \subseteq \operatorname{Van}(H)$.

Now, if $x \in H \backslash L$, then $x$ does not centralize some chief factor $V_{i}$ and, by the previous paragraphs, we conclude that $x \in \operatorname{Van}(H)$. So, $H \backslash L \subseteq \operatorname{Van}(H)$, and by Lemma 2.1, we also have that $G \backslash L P \subseteq \operatorname{Van}(G)$.

(v) $L P \backslash F P \subseteq \operatorname{Van}(G)$.

We first observe that if $N / F$ is a normal subgroup of $H / F, q$ is an odd prime dividing $|N / F|$ and there exists a character $\psi \in \operatorname{Irr}(N / F)$ of $q$-defect zero, then every Sylow $q$-subgroup $Q$ of $N$ is cyclic. In fact, $\psi$ vanishes on every element of $N / F$ having order divisible by $q$. So, by Lemma 2.1 and Lemma 2.2, $Q F \backslash$ $F \subseteq \operatorname{Van}(H)$, and again by Lemma 2.1 $Q F P \backslash F P \subseteq \operatorname{Van}(G)$. Since step (iii) yields $F P \backslash P \subseteq \operatorname{Van}(G)$, it follows that $Q F P \backslash P \subseteq \operatorname{Van}(G)$ and, in particular, $Q P \backslash P \subseteq \operatorname{Van}(G)$. As no vanishing element of $G$ has order divisible by $p q$, this implies that $Q$ acts fixed-point freely on $P$. Since $q$ is odd, $Q$ must be cyclic.

If $L=F$, then $(v)$ clearly holds. So, we may assume that $L>F$. Let $M / F$ be a minimal normal subgroup of $H / F$ contained in $L / F$. We first prove that $M / F$ is nonabelian. If this is not the case, then $M / F$ is a $t$-group for some prime $t$. Write $R=\mathbf{O}_{t^{\prime}}(F) \unlhd H$ and let $T$ be a Sylow $t$-subgroup of $M$. By the definition of $L$, we see that $T$ acts trivially on the terms of an $H$-chief series of $F$. Thus, $T$ acts trivially on the terms of an $H$-chief series of $R$ and hence, by coprimality, $T$ centralizes $R$. This yields $T \leq \mathbf{O}_{t}(M) \leq \mathbf{O}_{t}(H) \leq F$, contradicting $M>F$.

Therefore, $M / F$ is the direct product of $m$ copies of a nonabelian simple group $S$, for a suitable positive integer $m$. Let now $q$ be a prime divisor of $|S|$, with $q \neq 2,3$ (such a $q$ does certainly exist, by Burnside's theorem). By Theorem 2.8, $S$ has an irreducible character of $q$-defect zero and then $M / F$ has an irreducible 
character of $q$-defect zero too. Hence, $M$ has cyclic Sylow $q$-subgroups and this clearly yields $m=1$, i.e. $M / F$ is a simple group.

We now prove the following

Claim: $M / F$ has irreducible characters of $q$-defect zero for every $q \in \pi(M / F)$.

Observe that, as can be checked in [2], the groups $M_{12}, M_{22}, M_{24}, J_{2}, H S$, $R u, C o_{1}, B M, A_{8}$ and $A_{9}$ all have irreducible characters of 3 -defect zero and noncyclic Sylow 3-subgroups. Similarly, Suz, $\mathrm{Co}_{3}$ and $A_{n}$ for every $n \geq 10$ all have irreducible characters of 5-defect zero (by Theorem 2.8) and noncyclic Sylow 5 -subgroups. Hence, $M / F$ is not isomorphic to any of the groups listed above. Taking into account Theorem 2.8, it only remains to exclude the possibility that $M / F$ is isomorphic to $A_{7}$.

Looking for a contradiction, assume $M / F \simeq A_{7}$, and define $D$ to be the smallest term of the derived series of $M$. Then $D=D^{\prime}$ (i.e. $D$ is perfect) and $M / D$ is solvable. Since $M / F$ is perfect, it follows that $M=F D$. In fact, $F D \unlhd M$ and $M / F D$ is a quotient of the perfect group $M / F$ and of the solvable group $M / D$, so $M / F D=1$. Let $Z=F \cap D$. Then $D / Z \simeq M / F \simeq A_{7}$.

We now show that $D$ centralizes $F$. Let $M_{j}$ be the largest term in the $H$-chief series $1=M_{0} \leq M_{1} \leq \cdots \leq M_{n}=F$ centralized by $D$. Assume, working for a contradiction, that $j<n$ and let $N=M_{j+1}$. Since $D \leq L$, we have that $[N, D] \leq M_{j}$. So $[N, D, D]=[D, N, D]=1$. Hence the Three Subgroup Lemma yields $[D, D, N]=1$, i.e. $\left[D^{\prime}, N\right]=[D, N]=1$. Therefore $D$ centralizes $N$, against the choice of $M_{j}$. It follows that $M_{j}=F$.

Hence, we see that $Z=D \cap F \leq \mathbf{Z}(D)$. Also, clearly $Z \leq D^{\prime}$ because $D$ is perfect. Thus, it follows that $D$ is a factor group of the Schur covering group $6 . A_{7}$ of $A_{7}$.

Let $T$ be the normalizer in $D$ of a subgroup of order 7. Clearly $|T|=21|Z|$. Checking in [2] the columns of the lifting orders corresponding to the conjugacy classes of the elements of order 3 in every factor group of $6 . A_{7}$, we see that $T$ contains a noncyclic subgroup of order 21 . We also see that there exists a character $\psi \in \operatorname{Irr}(D)$ of degree 21 vanishing on $T^{y} \backslash Z$ for every $y \in H$. So, Lemma 2.1 and Lemma 2.2 yield that $T P \backslash Z P \subseteq \operatorname{Van}(G)$. By step $(i)$, we also have $Z P \backslash P \subseteq$ $\operatorname{Van}(G)$. Therefore $T P \backslash P \subseteq \operatorname{Van}(G)$. Since no vanishing element in $G$ has order divisible by $p q$, for every $q \neq p$, we see that $T$ acts fixed-point freely on $P$. It follows that $T P$ is a Frobenius group. But $T$, as a Frobenius complement of order $21|Z|$, must have cyclic subgroups of order $3 \cdot 7$, a contradiction. This shows that $M / F$ is not isomorphic to $A_{7}$ and concludes the proof of the claim.

As a consequence of the Claim, recalling Lemma 2.7 and Lemma 2.1, we get that $M \backslash F \subseteq \operatorname{Van}(H)$. Hence, again by Lemma 2.1, $M P \backslash F P \subseteq \operatorname{Van}(G)$. As step (iii) yields $F P \backslash P \subseteq \operatorname{Van}(G)$, we see that $M P \backslash P \subseteq \operatorname{Van}(G)$. Since $G$ has no vanishing element of order divisible by $p q$ for every $q \neq p$, the group $M P$ is a Frobenius group with complement $M$. This implies $M / F \simeq A_{5}$.

We have hence proved that if $M / F$ is a minimal normal subgroup of $H / F$, with $M / F \leq L / F$, then $M / F \simeq A_{5}$. In particular, if $M_{1} / F, M_{2} / F$ are distinct minimal normal subgroups of $H / F$ contained in $L / F$, then $M_{1} M_{2} / F \simeq A_{5} \times A_{5}$ has characters of 5-defect zero. This implies (by the first paragraph in the proof of $(v)$ ) that the Sylow 5-subgroups of $M_{1} M_{2}$ are cyclic, a contradiction.

Therefore, the socle of $L / F$ is simple, isomorphic to $A_{5}$. So $L / F$ is isomorphic to either $A_{5}$ or $S_{5}$. In both cases, we see by direct inspection that every nontrivial 
element of $L / F$ is in $\operatorname{Van}(L / F)$. So, by Lemma 2.1 and Lemma 2.2, we get that $L P \backslash F P \subseteq \operatorname{Van}(G)$.

Conclusion. By steps (iii), (iv) and $(v)$, we have $G \backslash P \subseteq \operatorname{Van}(G)$. Hence, no element of $G \backslash P$ has order divisible by $p$ and we get $\mathbf{C}_{G}(x) \leq P$, for every $x \in P$ with $x \neq 1$. Therefore, $G$ is a Frobenius group with kernel $P$ and complement $H$.

To conclude the proof, we now drop the assumption that $\mathbf{O}_{p^{\prime}}(G)=1$ and show that $K=\mathbf{O}_{p^{\prime}}(G)$ is nilpotent. Observe first that, as $P>1$, Proposition 2.5 yields $K \cap \operatorname{Van}(G)=\emptyset$. Next, note that the Fitting subgroup $\mathbf{F}(G)$ of $G$ is contained in $P K$, because $\mathbf{F}(G / K)=P K / K$. Setting $M=\mathbf{F}(G) \cap K$, our aim is to show that $M=K$. Assume this is not the case, and take a minimal normal subgroup $N / M$ of $G / M$ with $N \leq K$. If $N / M$ is nonabelian then, by Corollary 2.9, we get $N / M \cap \operatorname{Van}(G / M) \neq \emptyset$. Thus, by Lemma $2.1, K \cap \operatorname{Van}(G) \neq \emptyset$, a contradiction. So $N / M$ is an abelian $q$-group for some prime $q$. Let $Q$ be a Sylow $q$-subgroup of $N$ and let $L=\mathbf{O}_{q^{\prime}}(N)$. Note that $N=Q L$. Clearly $L$ is centralized by $Q \cap M$, but not by $Q$, as otherwise $Q$ would be contained in $\mathbf{O}_{q}(N) \leq \mathbf{F}(G)$ and hence $N \leq M$, against the choice of $N$. Thus, by coprimality, there exists a $G$-chief factor $V=A / B$, where $A, B \leq L$, which is not centralized by $Q$. It follows that $\mathbf{C}_{N}(V)<N$. Recalling that the nilpotency of $M$ yields $M \leq \mathbf{C}_{N}(V)$, we are in a position to apply Lemma 2.3, obtaining $N \backslash \mathbf{C}_{N}(V) \subseteq \operatorname{Van}(G)$. This leads to the final contradiction $K \cap \operatorname{Van}(G) \neq \emptyset$.

Theorem C, which we state again, follows immediately from Theorem 3.1.

Theorem C. Let $G$ be a group, $p$ a prime divisor of $|G|$, and $P$ a Sylow $p$-subgroup of $G$. If $G$ does not have any vanishing element of order divisible by $p$, then the following conclusions hold:

(a) $P$ is normal in $G$;

(b) either $G$ is abelian, or $G / \mathbf{O}_{p^{\prime}}(G)$ is a Frobenius group with kernel $P \mathbf{O}_{p^{\prime}}(G) / \mathbf{O}_{p^{\prime}}(G)$, and $\mathbf{O}_{p^{\prime}}(G)$ is nilpotent.

Proof. Part (a) is [3, Corollary 3]. In order to prove Part (b), we apply Theorem 3.1. If $G / \mathbf{O}_{p^{\prime}}(G)$ is a $p$-group, then by (a) we have that $G$ is nilpotent. By Corollary 2.6 and Burnside's theorem on zeros of nonlinear irreducible characters, it follows that $G$ is abelian.

It is worth mentioning that there actually exist groups $G$ and primes $p$ satisfying the assumptions of Theorem $\mathrm{C}$ and such that $\mathbf{O}_{p^{\prime}}(G)$ is nonabelian. For instance, take a Hall $\{2,7\}$-subgroup of the Suzuki group $\operatorname{Suz}(8)$ and a cyclic group $P$ of order 29. Consider the nontrivial action of $H$ on $P$ and the corresponding semidirect product $G$. One can check that $G$ has no vanishing elements of order divisible by 29 (in fact $\operatorname{Van}(G)$ consists just of elements of order 7 ), and $\mathbf{O}_{29^{\prime}}(G)$ is nonabelian.

\section{Some Applications of Theorem 3.1}

Our aim in this section is to derive some consequences of Theorem 3.1 (and of its corollary Theorem C). We shall use them in the proof of Theorem B.

Proposition 4.1. Assume that, for a given prime $p$, the group $G$ has a normal Sylow p-subgroup $P$ and that $p$ is an isolated vertex of $\Gamma(G)$. Then $\Gamma(G)$ has at most two connected components and, if $G$ is nonsolvable, then $G$ has a unique nonabelian composition factor $S$ and $S \simeq A_{5}$. 
Proof. The vertex $p$ is isolated in $\Gamma(G)$ exactly when $\operatorname{Van}(G)$ contains elements of order divisible by $p$, but no element of order divisible by $p q$ for every prime $q \neq p$. We can hence apply Theorem 3.1. Note also that, as $P \cap \operatorname{Van}(G) \neq \emptyset$, Proposition 2.5 implies that $\mathbf{O}_{p^{\prime}}(G)=1$. So, either $G=P$ and $\Gamma(G)$ consists of the single vertex $p$, or $G$ is a Frobenius group and we are done by Lemma 2.11.

Note that, as the group $A_{7}$ shows (choosing any prime divisor of $\left|A_{7}\right|$ as the prime $p$ ), the assumption concerning the normality of a Sylow $p$-subgroup is essential in the above statement.

Proposition 4.2. Let $G$ be a group, and let $\sigma$ be the vertex set of $\Gamma(G)$. Assume $\sigma \neq \pi(G)$. Then $\Gamma(G)$ is connected. Moreover, if $G$ is nonsolvable, then $G$ has a unique nonabelian composition factor $S$ and $S \simeq A_{5}$.

Proof. Set $\rho=\pi(G) \backslash \sigma$. Our assumption guarantees that $\rho \neq \emptyset$, and Part (a) of Theorem $\mathrm{C}$ applied to each prime in $\rho$ yields that $G$ has a nilpotent normal Hall $\rho$-subgroup. In particular, for a given $p$ in $\rho$, the group $\bar{G}=G / \mathbf{O}_{p^{\prime}}(G)$ is a $(\sigma \cup\{p\})$-group. Also, by Part (b) of Theorem C, $\mathbf{O}_{p^{\prime}}(G)$ is nilpotent and either $\bar{G}$ is a Frobenius group or $G$ is abelian. If $G$ is abelian, then $\Gamma(G)$ is the empty graph and the result clearly holds. Therefore we may assume that $\bar{G}$ is a Frobenius group. If $G$ is nonsolvable, then $\bar{G}$ is nonsolvable and hence $G$ has a unique nonabelian composition factor, which is isomorphic to $A_{5}$.

It remains to prove that $\Gamma(G)$ is connected. Let $\bar{H}$ be a Frobenius complement of $\bar{G}$. Then, by Lemma 2.11 , all the primes in $\pi(\bar{H})$ are vertices in the same connected component of $\Gamma(\bar{G})$. Hence, it suffices to show that every prime $q \in \sigma \backslash \pi(\bar{H})$ is adjacent to a prime in $\pi(\bar{H})$. The prime $q$ is a vertex of $\Gamma(G)$, thus there exists $g \in \operatorname{Van}(G)$ of order divisible by $q$. As $p \in \rho$, the prime $p$ does not divide $o(g)$. If there is no prime $r \in \pi(\bar{H})$ dividing $o(g)$, then $\left(\left|G: \mathbf{O}_{p^{\prime}}(G)\right|, o(g)\right)=1$ and $g \in \mathbf{O}_{p^{\prime}}(G)$. Let now $P$ be a Sylow $p$-subgroup of $G$. We know that $P$ is normal in $G$, and clearly $\mathbf{O}_{p^{\prime}}(G) \cap P=1$. Since $g$ lies in $\mathbf{O}_{p^{\prime}}(G) \cap \operatorname{Van}(G)$, by Proposition 2.5 we get $g x \in \operatorname{Van}(G)$ for every $x \in P$. Choosing $x \neq 1$, we get a vanishing element of order divisible by $p$, a contradiction. Therefore $o(g)$ is divisible by some prime $r \in \pi(\bar{H})$, which shows that $q$ is adjacent to $r$ in $\Gamma(G)$, as desired.

\section{Proof of Theorem A And Theorem B}

In what follows, we shall denote by $V(\mathcal{G})$ the vertex set of a graph $\mathcal{G}$, and by $\mathrm{n}(\mathcal{G})$ the number of connected components of $\mathcal{G}$.

As a last preliminary result, we gather some information on $\Gamma(G)$ when $G$ is an extension of a $p$-group by $A_{7}$.

Lemma 5.1. Let $G$ be a group, and $N$ a nontrivial normal subgroup of $G$. If $N$ is a p-group and $G / N \simeq A_{7}$, then $V(\Gamma(G))=\pi(G)$ and $\Gamma(G)$ has at most two connected components.

Proof. Recalling Lemma 2.1(a), by factoring out a suitable normal subgroup of $G$ we can assume that $N$ is minimal normal in $G$.

The graph $\Gamma\left(A_{7}\right)$ has four vertices (namely 2, 3, 5 and 7 ), whence it will be enough to show that there are three distinct primes $q_{1}, q_{2}, q_{3} \in\{2,3,5,7\}$ that are connected to $p$ in $\Gamma(G)$. In order to prove that a prime $q \neq p$ is adjacent to $p$ in $\Gamma(G)$, we shall argue that there exists a $p^{\prime}$-element $x=g N \in \operatorname{Van}(G / N)$ of order 
divisible by $q$ and such that $\mathbf{C}_{N}(x) \neq 1$. Then, taking a nontrivial $y \in \mathbf{C}_{N}(x)$, the element $g y$ has order divisible by $p q$ and $g y \in g N \subseteq \operatorname{Van}(G)$ by Lemma 2.1.

Since the dimension of the eigenspace of the eigenvalue 1 is invariant by field extensions, considering also that the dimensions of eigenspaces add up in a direct sum of modules, we can reduce to the case that $N$ is an absolutely irreducible $A_{7}$-module. So, we refer to the character tables of $A_{7}$ in [12].

If $p \neq 2,3,5,7$, then for every $\phi \in \operatorname{IBr}_{\mathrm{p}}\left(\mathrm{A}_{7}\right)=\operatorname{Irr}\left(\mathrm{A}_{7}\right)$ we have $\left\langle\phi, 1_{\langle x\rangle}\right\rangle \neq 0$, for $x=(1,2,3)(4,5,6), x=(1,2,3,4,5)$ and $x=(1,2)(3,4,5,6)$.

If $p=3$, then $\left\langle\phi, 1_{\langle x\rangle}\right\rangle \neq 0$ for every $\phi \in \operatorname{IBr}_{\mathrm{p}}\left(\mathrm{A}_{7}\right)$ when $x=(1,2,3,4,5)$ and $x=(1,2)(3,4,5,6)$.

If $p=5$, then $\left\langle\phi, 1_{\langle x\rangle}\right\rangle \neq 0$, for every $\phi \in \operatorname{IBr}_{\mathrm{p}}\left(\mathrm{A}_{7}\right)$ when $x=(1,2,3)(4,5,6)$ or $x=(1,2)(3,4,5,6)$.

If $p=7$, then $\left\langle\phi, 1_{\langle x\rangle}\right\rangle \neq 0$, for every $\phi \in \operatorname{IBr}_{\mathrm{p}}\left(\mathrm{A}_{7}\right)$ when $x=(1,2,3)(4,5,6)$, $x=(1,2)(3,4,5,6)$ or even $x=(1,2,3,4,5)$.

If $p=2$, then $\left\langle\phi, 1_{\langle x\rangle}\right\rangle \neq 0$, when $\phi(1) \neq 6$ and $x=(1,2,3)(4,5,6)$ or $x=$ $(1,2,3,4,5,6,7)$ or when $\phi(1)=6$ and $x=(1,2,3)(4,5,6)$ or $x=(1,2,3,4,5)$.

For the sake of completeness, it may be worth mentioning that there are groups satisfying the assumptions of the above lemma, and whose vanishing prime graph is disconnected. An example of this kind can be constructed as follows. Let $H$ be an irreducible subgroup of $\mathrm{GL}(6,2)$ isomorphic to $A_{7}$, and let $V$ be the natural module for GL(6,2). Set $G$ to be the semidirect product $V \rtimes H$ formed according to the natural action. It is possible to check that $\Gamma(G)$ has two connected components: the prime 7 is an isolated vertex, whereas 2,3 and 5 are pairwise adjacent.

We are now in a position to prove Theorem B, which we state again.

Theorem B. Let $G$ be a nonsolvable group. If $\Gamma(G)$ is disconnected, then $G$ has a unique nonabelian composition factor $S$, and $\mathrm{n}(\Gamma(G)) \leq \mathrm{n}(\Pi(S))$ unless $G$ is isomorphic to $A_{7}$.

Proof. As $\Gamma(G)$ is disconnected, Proposition 4.2 yields that $V=V(\Gamma(G))$ coincides with $\pi(G)$. We argue by induction on the order of the group. Recall that, if $N$ is a normal subgroup of $G$, then $\Gamma(G / N)$ is a subgraph of $\Gamma(G)$.

Assume first that $G$ has nontrivial solvable normal subgroups. So $N=\mathbf{O}_{p}(G) \neq$ 1 for some prime $p$. In view of Lemma 5.1 we are done if $G / N \simeq A_{7}$. So, we may assume this is not the case.

Suppose $V(\Gamma(G / N))=\pi(G / N)$. If $\Gamma(G / N)$ is connected, then $N$ must be a Sylow $p$-subgroup of $G$ and $p$ an isolated vertex of $\Gamma(G)$, as $\Gamma(G)$ is disconnected. But then by Proposition 4.1 we see that $G$ has just one nonabelian composition factor $S$ and that $\mathrm{n}(\Gamma(G)) \leq 2<3=\mathrm{n}(\Pi(S))$, as $S \simeq A_{5}$. If $\Gamma(G / N)$ is disconnected, then induction yields that $G / N$, and hence $G$, has a unique nonabelian composition factor $S$ and that $\mathrm{n}(\Gamma(G / N)) \leq \mathrm{n}(\Pi(S))$. Now, $\mathrm{n}(\Gamma(G / N))=\mathrm{n}(\Gamma(G))$ unless $N$ is a Sylow subgroup of $G$ and $p$ is an isolated vertex of $\Gamma(G)$. But also in this case, again by Proposition 4.1, we get $\mathrm{n}(\Gamma(G / N))=\mathrm{n}(\Gamma(G))=2$.

Suppose now $V(\Gamma(G / N)) \neq \pi(G / N)$. By Proposition 4.2, $\Gamma(G / N)$ is connected, and $G / N$ (whence $G$ ) has a unique nonabelian composition factor $S$, with $S \simeq A_{5}$. In particular, $\mathrm{n}(\Pi(S))=3$.

Set $\pi=\pi(G / N) \backslash V(\Gamma(G / N))$, and observe that $p$ cannot lie in $\pi$ by Part (a) of Theorem C. By the same result, the group $G / N$ has a nilpotent normal Hall 
$\pi$-subgroup $K / N$. Denoting by $H$ a complement for $N$ in $K$, we get that $H$ is a Hall $\pi$-subgroup of $G$. Set $M=\mathbf{C}_{H}(N)$. We claim that $M$ is a normal subgroup of $G$. In fact, $M=\mathbf{C}_{K}(N) \cap H$ is a Hall $\pi$-subgroup of $\mathbf{C}_{K}(N)$, whereas $\mathbf{Z}(N)=\mathbf{C}_{K}(N) \cap N$ is a Sylow $p$-subgroup of $\mathbf{C}_{K}(N)$. So $\mathbf{C}_{K}(N)=M \times \mathbf{Z}(N)$ and hence $M=\mathbf{O}_{p^{\prime}}\left(\mathbf{C}_{K}(N)\right)$. Therefore $M$, which is characteristic in $\mathbf{C}_{K}(N) \unlhd G$, is normal in $G$. We shall consider the two possibilities $M=H$ and $M \neq H$.

Assume $M=H$. Now, $H$ and $N$ are nilpotent normal subgroups of $G$, so that $K=H N$ is also nilpotent. If $\operatorname{Van}(G) \cap K \neq \emptyset$, then Corollary 2.6 yields an element $g \in \operatorname{Van}(G) \cap K$ whose order is divisible by every prime in $\pi(K)$. In particular, the elements in $\pi(K)=\pi \cup\{p\}$ are in the same connected component of $\Gamma(G)$. So, $\mathrm{n}(\Gamma(G)) \leq \mathrm{n}(\Gamma(G / N))+1 \leq 2<\mathrm{n}(\Pi(S))$. On the other hand, assume $\operatorname{Van}(G) \cap K=\emptyset$, and let $q$ be in $\pi(K)$. Since $V=\pi(G)$, there exists $g \in \operatorname{Van}(G)$ such that $o(g)$ is a multiple of $q$. As $g \notin K$, we get that $o(g)$ is divisible by some prime in $\pi(G / K)=V(\Gamma(G / N))$. This proves that every element in $\pi(K)$ is adjacent to some vertex in $V(\Gamma(G / N))$, thus we get $\mathrm{n}(\Gamma(G))=\mathrm{n}(\Gamma(G / N))=1$.

Now, we may assume $M \neq H$. Let $Z / M N=\mathbf{Z}(K / M N) \unlhd G / M N$. We have that $(Z / M) /(M N / M) \simeq Z / M N$ is abelian. We claim that $\mathbf{C}_{Z / M}(M N / M) \leq$ $M N / M$. Write $C / M=\mathbf{C}_{Z / M}(M N / M)$. Since $[C / M, M N / M]=1$, we get $[C, M N] \leq M$. Therefore, $[C, N] \leq M \cap N=1$. Hence $C \leq \mathbf{C}_{K}(N) \leq M N$, as we wanted. Note also that, $K / N$ being a $p^{\prime}$-group, we get $(|Z / M N|,|M N / M|)=1$.

By Lemma 2.4 and Lemma 2.1, we have that $Z \backslash M N \subseteq \operatorname{Van}(G)$. Recalling now that $K / N$ is nilpotent, and that $Z / M N$ is the center of the nontrivial nilpotent group $K / M N$, on one hand we get $\pi(Z / M N)=\pi(K / M N)$, whence $\pi(Z / N)=$ $\pi(K / N)$; on the other hand, since $Z / N$ is nilpotent, there exists $z N \in(Z / N) \backslash$ $(M N / N)$ such that $o(z N)$ is divisible by every prime in $\pi(Z / N)$. Therefore $z \in$ $Z \backslash M N \subseteq \operatorname{Van}(G)$ has order divisible by every prime in $\pi=\pi(K / N)$, which implies that all such primes lie in the same connected component of $\Gamma(G)$. Our discussion so far yields that each connected component of $\Gamma(G)$ is a union of some of the following three sets: $\{p\}, \pi$, and $V(\Gamma(G / N))$. Hence, $\mathrm{n}(\Gamma(G)) \leq 3=\mathrm{n}(\Pi(S))$.

Therefore, for the rest of the proof we shall assume that $G$ has no solvable normal subgroup.

Let $M$ be a minimal normal subgroup of $G$.

Claim. every $p \in V$ is connected in $\Gamma(G)$ to some $q \in \pi(M)$.

This is trivially true if $p \in \pi(M)$, so we can assume that $p$ does not divide $|M|$. If $p \in V(\Gamma(G / M))$, then there exists an element $x M \in \operatorname{Van}(G / M)$ such that $p$ divides the order of $x$. As $M$ in nonsolvable, the main result of [15] implies that there exists a nontrivial $y \in \mathbf{C}_{M}(x)$. But then $x y \in x M \subseteq \operatorname{Van}(G)$, and hence $p$ is connected to every prime divisor of $o(y)$ in $\Gamma(G)$. Finally, if $p \in \pi(G / M) \backslash V(\Gamma(G / M))$, then Theorem $\mathrm{C}$ implies that $G / M$ has a normal Sylow $p$-subgroup $P / M$, whence there exists a subgroup $Z \unlhd G$ such that $|Z / M|=p$. Let $\langle x\rangle$ be a complement for $M$ in $Z$. Observe that $\mathbf{C}_{Z}(M) \leq M$, as otherwise $x \in \mathbf{O}_{p}(Z) \leq \mathbf{O}_{p}(G)=1$, a contradiction. Thus, $Z \backslash N \subseteq \operatorname{Van}(G)$ by Lemma 2.4. On the other hand, by Thompson's nilpotency criterion there must exist a nontrivial $y \in \mathbf{C}_{M}(x)$. Now, $x y \in \operatorname{Van}(G)$ and hence $p$ is connected to every prime divisor of $o(y)$ in $\Gamma(G)$. The claim is proved.

We now show that $M$ is a simple group. If this is not the case then, by Corollary 2.9, every $q \in \pi(M), q \geq 5$, is adjacent to every other $q^{\prime} \in \pi(M)$ in $\Gamma(G)$. Recalling that $\pi(M) \subseteq V$, then all primes in $\pi(M)$ lie in the same connected 
component of $\Gamma(G)$ and, by the Claim, this implies that $\Gamma(G)$ is connected, a contradiction.

Moreover, $\mathbf{C}_{G}(M)=1$. If not, then there exists a minimal normal subgroup $N$ of $G$ such that $N \cap M=1$. Since $N$ is nonsolvable as well, by Corollary 2.9 we know that there exists a $g \in N \cap \operatorname{Van}(G)$. Now, by Proposition 2.5 all products $g m$, with $m \in M$, are vanishing elements of $G$. Hence every prime in $\pi(M)$ is connected to every single prime divisor of $g$ in $\Gamma(G)$. By the Claim, this again implies that $\Gamma(G)$ is connected, against our assumption.

Therefore, $M$ is the socle of $G$ and, as $M$ is simple, $G$ is an almost simple group. In particular, $G / M$ is solvable (by Schreier's conjecture) and $M$ is the only nonabelian composition factor of $G$.

Set $\sigma=\pi(M)$. By Corollary 2.9, if $p, q \in \sigma$ are adjacent in $\Pi(M)$ then they are adjacent in $\Gamma(G)$ as well, unless $\{p, q\}=\{2,3\}$. Therefore, recalling that $\sigma \subseteq V$, the subgraph induced by $\Gamma(G)$ on $\sigma$ is either $\Pi(M)$ or $\Pi(M)$ with the edge $\{2,3\}$ deleted. Taking also into account Lemma 2.7 and Theorem 2.8, the latter situation can occur only if $M$ belongs to a list consisting of some sporadic and alternating groups. However, Proposition 2.10 yields that, unless $M \simeq A_{7}$, there exist $g \in M$ and $\chi \in \operatorname{Irr}(\operatorname{Aut}(M))$ such that $o(g)=6$, the restriction of $\chi$ to $S$ is irreducible, and $\chi(g)=0$. As a consequence, the subgraph induced by $\Gamma(G)$ on $\sigma$ actually coincides with $\Pi(M)$ unless $M \simeq A_{7}$. In view of the Claim, we now get $\mathrm{n}(\Gamma(G)) \leq \mathrm{n}(\Pi(M))$ if $M \not A_{7}$. On the other hand, assume $M \simeq A_{7}$ : since we are excluding the possibility $G \simeq A_{7}$, we only have to check the case $G \simeq S_{7}$, and direct inspection shows that $\mathrm{n}\left(\Gamma\left(S_{7}\right)\right)=2<3=\mathrm{n}\left(\Pi\left(A_{7}\right)\right)$. This concludes the proof.

Finally we are ready to prove Theorem A, which we state again.

Theorem A. Let $G$ be a group. Then $\Gamma(G)$ has at most six connected components.

Proof. If $G$ is solvable, then $\mathrm{n}(\Gamma(G)) \leq 2$ by Theorem A of [4]. If $G$ is nonsolvable, we use Theorem B and the fact that, as shown in [8] and [16], $\mathrm{n}(\Pi(S))$ is at most 6 when $S$ is a nonabelian simple group.

\section{6. $\Gamma(G)$ AND $\Pi(G)$ : Similarities AND DifFERENCES}

The purpose of this final section is to discuss some aspects of the relationship between the graphs $\Pi(G)$ and $\Gamma(G)$. As we shall see, Theorem A and Theorem B hold also replacing $\Gamma(G)$ with $\Pi(G)$ (see Theorem 6.3 and Theorem 6.2 respectively; the latter actually differs from Theorem B on the fact that $A_{7}$ doesn't need to be excluded). Moreover, Proposition 6.4 states that the two graphs are actually the same when $G$ is a nonabelian simple group other than $A_{7}$.

Nevertheless, Example 6.5 shows that the two graphs are in general not so similar, in the sense that the edge set of $\Gamma(G)$ can be "much smaller" than that of $\Pi(G)$, even if the vertex sets are the same. Therefore, the fact that the bound for the number of connected components is the same for the two graphs appears to be not obvious in principle.

Theorem 6.2 can be deduced from Theorem A and Lemma 1-Lemma 3 in [16]. Here, we present a short proof. We first prove an auxiliary result.

Lemma 6.1. Let $N$ be a nonnilpotent normal subgroup of the group $G$. Then the following conclusions hold.

(a) $\mathrm{n}(\Pi(G)) \leq \mathrm{n}(\Pi(N))$. 
(b) If $\mathbf{C}_{G}(N) \neq 1$, then $\Pi(G)$ is connected.

Proof. Let $p \in \pi(G) \backslash \pi(N)$ and let $x \in G$ be an element of order $p$. Since $N$ is not nilpotent, by Thompson's nilpotency criterion there exists a nontrivial element $y \in \mathbf{C}_{N}(x)$. So, the vertex $p$ of $\Pi(G)$ is connected to all prime divisors of $o(y)$. This yields (a).

Assume now $\mathbf{C}_{G}(N) \neq 1$ and choose $q \in \pi\left(\mathbf{C}_{G}(N)\right)$. Then every prime divisor of $|N|$ is connected to $q$ in $\Pi(G)$. As by the previous paragraph every prime in $\pi(G)$ is connected to a prime in $\pi(N)$, it follows that $\Pi(G)$ is connected.

Theorem 6.2. Let $G$ be a nonsolvable group. If $\Pi(G)$ is disconnected, then $G$ has a unique nonabelian composition factor $S$ and $\mathrm{n}(\Pi(G)) \leq \mathrm{n}(\Pi(S))$.

Proof. Let $N$ be a minimal normal subgroup of $G$.

Assume first that $N$ is solvable, hence $N$ is an elementary abelian $p$-group for a suitable prime $p$. For this case, we argue by induction on the order of the group. If $p$ is not connected to any $q \in \pi(G / N)$, then $N$ is a Sylow $p$-subgroup of $G$ and $p$ does not divide $o(g)$ for all $g \in G \backslash N$. In this situation, $G$ is a Frobenius group (with kernel $N$ ), and hence $\mathrm{n}(\Pi(G))=2$ by Lemma 2.11. Moreover, we get $S \simeq A_{5}$, whence $\mathrm{n}(\Pi(G))=2<3=\mathrm{n}(\Pi(S))$. Therefore we can assume that $p$ is connected in $\Pi(G)$ to some vertex of $\Pi(G / N)$. As $\Pi(G / N)$ is a subgraph of $\Pi(G)$ and $\Pi(G)$ is disconnected, then $\Pi(G / N)$ is disconnected as well, and we are done by induction.

Next, assume that $N$ is nonsolvable. As $\Pi(N)$ is clearly connected when $N$ is not simple, Lemma 6.1 implies that $N$ is simple and that $\mathbf{C}_{G}(N)=1$. Hence $G$ is an almost simple group, with socle $N$. Since now $G / N$ is isomorphic to a subgroup of the solvable group $\operatorname{Out}(N)$ (here we use Schreier's conjecture), we see that $N$ is the unique nonabelian composition factor of $G$. Finally, $\mathrm{n}(\Pi(G)) \leq \mathrm{n}(\Pi(N))$ by (a) of Lemma 6.1.

We can now state the following result (see [16]).

Theorem 6.3. Let $G$ be a group. Then $\Pi(G)$ has at most six connected components.

Proof. If $G$ is solvable, then $\mathrm{n}(\Pi(G)) \leq 2$ (see [16, pag. 487]). If $G$ is nonsolvable and $\Pi(G)$ is disconnected, we use Theorem 6.2 and the fact, proved in [8] and [16], that $\mathrm{n}(\Pi(S)) \leq 6$ for every finite simple group $S$.

Let $S$ be a nonabelian simple group. Note that, by Part (a) of Theorem C, the graphs $\Gamma(S)$ and $\Pi(S)$ have the same vertex set. Also, taking into account Lemma 2.7, Theorem 2.8 and Proposition 2.10, the following proposition can be easily deduced.

Proposition 6.4. Let $S$ be a nonabelian simple group. Then $\Gamma(S)=\Pi(S)$, unless $S \simeq A_{7}$.

As already mentioned, the above result cannot be extended to include $A_{7}$. In fact, $\{2,3\}$ is an edge of $\Pi\left(A_{7}\right)$, but not of $\Gamma\left(A_{7}\right)$. Although, as we shall see in Example 6.5, there is a whole family of groups $G$ whose graphs $\Gamma(G)$ and $\Pi(G)$ have the same vertex set but fairly different edge sets, at the time of this writing the authors do not know of any example of a nonsolvable group $G \not A_{7}$ such that $V(\Gamma(G))=V(\Pi(G))$ and $\mathrm{n}(\Gamma(G)) \neq \mathrm{n}(\Pi(G))$. 
In contrast to what happens in the graph $\Pi(G)$, it was proved in [4, Theorem B] that the independence number (i.e. the maximal size of a set of pairwise nonadjacent vertices) of $\Gamma(G)$ can be arbitrarily large if $G$ is a solvable group. In particular, the edge set of $\Gamma(G)$ can be considerably smaller than the edge set of $\Pi(G)$ in the context of solvable groups. We give here a nonsolvable example.

Example 6.5. Let $k \geq 2$ be a natural number. Let $p_{1}, \ldots, p_{k}$ be distinct primes with $p_{i} \geq 7$. Set $n=\prod_{i} p_{i}$. Using Dirichlet's theorem on primes in arithmetic progression, pick $q_{1}, \ldots, q_{k}$ distinct primes such that $q_{j} \equiv 1 \bmod p_{i}$ for every $j \neq i$, $q_{j} \equiv 1 \bmod 5$ and $q_{j}>120 \cdot n$ for every $j$. Set $m=\prod_{i} q_{i}$.

Let $V_{j}$ be the additive group of the finite field $\operatorname{GF}\left(q_{j}^{2}\right)$ and $V=V_{1} \times \cdots \times V_{k}$. Set $H=C_{n} \times \operatorname{SL}(2,5)$, where $C_{n}$ is a cyclic group of order $n$. Denote by $C_{p_{i}}$ the subgroup of order $p_{i}$ of $C_{n}$. Next, we define a group action of $H$ on $V$. For each $i$, let $\lambda_{i}$ be an element of order $n / p_{i}$ of the multiplicative group of $\operatorname{GF}\left(q_{i}^{2}\right)$ and note that the right multiplication by $\lambda_{i}$ in $V_{i}$ defines an automorphism of order $n / p_{i}$. Therefore, this yields an action of $C_{n}$ on $V_{i}$ with kernel $C_{p_{i}}$. Also, by construction, $C_{n} / C_{p_{i}}$ acts fixed-point freely on $V_{i}$. Since $q_{i}^{2} \equiv 1 \bmod 5$, the group $\operatorname{Aut}\left(V_{i}\right)=\mathrm{GL}\left(2, q_{i}\right)$ contains a subgroup isomorphic to $\operatorname{SL}(2,5)$ acting fixed-point freely on $V_{i}$ (see $\left.[7, \mathrm{~V} .8 .8 \mathrm{~b})\right]$ ). As it commutes with the scalar action defined by $C_{n}$, this defines an action of $H$ on $V_{i}$, for each $i$, and so an action of $H$ on $V$.

Set $G=V \rtimes H$. Clearly, the vertex set of $\Pi(G)$ is $\left\{2,3,5, p_{i}, q_{i} \mid i=1, \ldots, k\right\}$. Also, since $\mathrm{SL}(2,5)$ acts fixed-point freely on $V$ and $C_{n} / C_{p_{i}}$ acts fixed-point freely on $V_{i}$, the edge set of $\Pi(G)$ is

$$
\left\{\left\{p_{i}, p_{j}\right\},\left\{q_{i}, q_{j}\right\},\left\{2, p_{i}\right\},\left\{3, p_{i}\right\},\left\{5, p_{i}\right\},\{2,3\},\{2,5\},\left\{p_{i}, q_{i}\right\} \mid 1 \leq i \neq j \leq k\right\} .
$$

We claim that $\Gamma(G)$ is the subgraph of $\Pi(G)$ obtained by deleting the edges $\left\{q_{i}, q_{j}\right\}, 1 \leq i \neq j \leq k$. This will show that $\left\{q_{1}, \ldots, q_{k}\right\}$ is an independent set in $\Gamma(G)$ and that the number of edges of $\Gamma(G)$ is considerably smaller than the number of edges of $\Pi(G)$. We start by noticing that $\operatorname{Van}(G) \cap V=\emptyset$. We argue by contradiction. Let $\chi$ be in $\operatorname{Irr}(G)$, and $g \in V$ such that $\chi(g)=0$. Now, $\chi_{V}=\lambda_{1}+\cdots+\lambda_{s}$, where $\lambda_{i}$ is an irreducible character of $V$ and $s$ divides $120 n$. In particular, since $\chi(g)=0$, we get a vanishing sum of $s m$-th roots of unity. Therefore, by the Main Theorem in [13], $s$ must be a linear combination with nonnegative integer coefficients of $q_{1}, \ldots, q_{k}$. Clearly, this contradicts the fact that we chose $q_{i}>120 n$ for every $i \in\{1, \ldots, k\}$. Next, we observe that no element of $G \backslash V$ has order divisible by more than one prime $q_{i}$. Since $\operatorname{Van}(G) \cap V=\emptyset$, we see that $\left\{q_{i}, q_{j}\right\}$ is not an edge of $\Gamma(G)$, for every $i \neq j$.

Recalling that every noncentral element of $\mathrm{SL}(2,5)$ is a vanishing element and that $\Gamma(H)$ is a subgraph of $\Gamma(G)$, by Proposition 2.5 we get that

$$
\left\{p_{i}, p_{j}\right\},\left\{2, p_{i}\right\},\left\{3, p_{i}\right\},\left\{5, p_{i}\right\},\{2,3\},\{2,5\}
$$

are edges of $\Gamma(G)$.

Consider, finally, the normal subgroup $N=V \rtimes C_{n}$ of $G$. Then $(|V|,|N / V|)=1$, $N / V$ is abelian and $\mathbf{C}_{N}(V)=V$. Hence, Lemma 2.4 yields that $N \backslash V \subseteq \operatorname{Van}(G)$. It follows that $\left\{p_{i}, q_{i}\right\}$ is an edge of $\Gamma(G)$, for every $1 \leq i \leq k$. 


\section{REFERENCES}

[1] D. Bubboloni, S. Dolfi, P. Spiga, Finite groups whose irreducible characters vanish only on p-elements, J. Pure and Applied Algebra 213 (2009) 370-376.

[2] J.H. Conway, R.T. Curtis, S.P. Norton, R.A. Parker, R.A. Wilson, Atlas of finite groups, Clarendon Press, Oxford, 1985.

[3] S. Dolfi, E. Pacifici, L. Sanus, P. Spiga, On the orders of zeros of irreducible characters, J. Algebra 321 (2009) 345-352.

[4] S. Dolfi, E. Pacifici, L. Sanus, P. Spiga, On the vanishing prime graph of solvable groups, to appear in J. Group Theory.

[5] A. Granville, K. Ono, Defect zero p-blocks for finite simple groups, Trans. Amer. Math. Soc. 348 (1996) 331-347.

[6] B. Huppert, Character Theory of Finite Groups, De Gruyter, Berlin, 1998.

[7] B. Huppert, Endliche Gruppen I, Springer, Berlin, 1983.

[8] N. Iiyori, H. Yamaki, Prime graph components of the simple groups of Lie type over the field of even characteristic, J. Algebra 155 (1993) 335-343.

[9] I.M. Isaacs, Character Theory of Finite Groups, Dover, New York, 1976.

[10] I.M. Isaacs, G. Navarro, T.R. Wolf, Finite group elements where no irreducible character vanishes, J. Algebra 222 (1999) 413-423.

[11] G. James, A. Kerber, The representation Theory of the Symmetric Group, Encyclopedia of Mathematics and its applications Vol. 16, Addison-Wesley Publishing Co. 1981.

[12] C. Jansen, K. Lux, R. Parker, R. Wilson, An atlas of Brauer characters, London Math. Soc. Monographs, New Series, 11. Oxford University Press, New York, 1995.

[13] T.Y. Lam, K.H. Leung, On vanishing sums of roots of unity, J. Algebra 224 (2000) 91-109.

[14] O. Manz, T.R. Wolf, Representations of solvable groups, Cambridge University Press, Cambridge, 1993.

[15] P. Rowley, Finite groups admitting a fixed-point-free automorphism group, J. Algebra 174 (1995) 724-727.

[16] J.S. Williams, Prime graph components of finite groups, J. Algebra 69 (1981) 487-513. 
Silvio Dolfi, Dipartimento di Matematica U. Dini,

Università degli Studi di Firenze, viale Morgagni 67/A, 50134 Firenze, Italy.

E-mail address: dolfi@math.unifi.it

Emanuele Pacifici, Dipartimento di Matematica F. Enriques, Università degli Studi di Milano, via Saldini 50, 20133 Milano, Italy.

E-mail address: emanuele.pacifici@unimi.it

Lucia Sanus, Departament d’Àlgebra, Facultat de Matemàtiques, Universitat de València, 46100 Burjassot, València, Spain.

E-mail address: lucia.sanus@uv.es

Pablo Spiga, Dipartimento di Matematica Pura ed Applicata, Università degli Studi di Padova, via Trieste 63, 35121 Padova, Italy.

E-mail address: spiga@math.unipd.it 